\title{
A New Approach to OTH Main Parameters Determination
}

\author{
Zenon SAAVEDRA ${ }^{1,2}$, Julio N. ARGOTA ${ }^{1}$, Miguel A. CABRERA ${ }^{1}$, Ana G. ELIAS ${ }^{2,3}$ \\ ${ }^{1}$ Laboratorio de Telecomunicaciones, Dpto. de Electricidad, Electrónica y Computación, Facultad de Ciencias Exactas y \\ Tecnología, Universidad de Tucumán (FACET-UNT), Av. Independencia 1800, 4000 Tucumán, Argentina \\ ${ }^{2}$ Consejo Nacional de Investigaciones Científicas y Técnicas, CONICET, Argentina \\ ${ }^{3}$ Laboratorio de Física de la Atmósfera, Dpto. de Física, FACET, UNT, INFINOA (CONICET-UNT), Av. Independencia \\ 1800, 4000 Tucumán, Argentina
}

zsaavedra@herrera.unt.edu.ar, nargota@herrera.unt.edu.ar, mcabrera@herrera.unt.edu.ar, aelias@herrera.unt.edu.ar

Submitted October 9, 2018 / Accepted May 27, 2019

\begin{abstract}
In this work, we propose a method based on a simulation that incorporates several models to provide the set of parameters needed on Over-The-Horizon radars (OTHR) performance evaluation, which consists in a versatile software tool. Obtaining the signals involved during transmission and reception is a complex and challenging task. Among them, the received signal is fundamental to design methods and algorithms in the target detection strategy. The parameters in the transmission and reception processes that define the radio link main features are determined in terms of target type, ionospheric conditions, radio link characteristics, and other environmental properties. The determination is done combining models to work assembled in a software tool that simulates the OTHR radio link. The tool gives the possibility of step away from the linear model, which uses mainly constant parameters and it is used commonly. A large number of set up parameters and also interconnections among several models enable to simulate nearer to actual search sceneries.
\end{abstract}

\section{Keywords}

OTH radar, clutter, ground range, MUF, radar cross section, ray tracing

\section{Introduction}

Over-the-Horizon radars (OTHR) are designed to detect targets which are beyond the horizon, or line of sight, using high frequency (HF) radio waves, which are bent back towards the Earth by the ionosphere [1], [2]. They are used regularly in early warning systems, maritime and airspace monitoring, upper atmosphere and ocean studies among others [2-4]. The most difference from conventional radars is that it requires real-time ionospheric conditions assessment and adaptive frequency selection as well. Physically, OTHR consists of a synchronous transmitter/receiver and an antenna array. To begin with, the transmission-reception process must be capable of constructing a beam, formed by the electromagnetic waves from each transmitting antenna, which propagates through the atmosphere, is bent back by the ionosphere, and reaches a target, which in turn, scatters and reemits part of this energy that propagates back, through the ionosphere and neutral atmosphere, to the receiving antennas.

Some transmitted parameters, such as amplitude, phase, and frequency, are affected by several factors which are crucial in the propagation process. These result in attenuation during wave propagation, energy loss at the reflection by the target, noise and clutter contamination. Figure 1 shows the physical processes involved in the transmission and reception that may modify radio signal characteristics in an OTHR system.

Some of the required parameters in HF band radars are the expected limits for the elevation angle and usable frequency, area coverage, noise and clutter levels, all of which are associated with given frequencies and time. These parameters strongly depend on the propagating media and radio link characteristics. In this paper, we propose a software tool that incorporates several models to provide the set of parameters needed to optimize the OTHR settings and signal processing later.

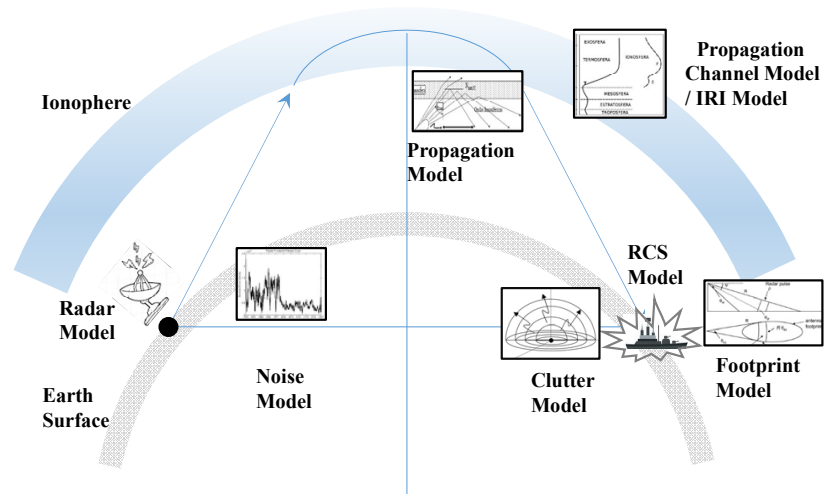

Fig. 1. Schematic diagram of the processes taking place during transmission and reception in an OTHR. 
In a comparison of the model here proposed with two other OTHR models described in [5] and [6], although they all contain several sub-models that allow defining each step in the transmission-reception process, the main differences between them and ours are: the use of a ionospheric model in our case instead of a constant ionosphere profile that allows for ionospheric variability, and the radar cross section that in our case is defined through electromagnetic simulation rather than an average value and a probability distribution function (PDF).

The sub-models depend on the ionosphere model used, and in our case, the use of a variable ionosphere results in output data nearer to an actual value from the search scenario. Certainly the ionosphere model must be able to exhibit the actual behavior of the terrestrial ionosphere.

The software has a set of interactions between its members (models). This allows manifesting variations in the output of any model according to its input but also on other models' output. Therefore, it admits defining and simulating different scenarios and also giving a first approximation to the actual conditions of the radio link.

\section{Methodology}

Each block in the process of transmission and reception in the software tool has a set of parameters which can be modified by the user. Unlike other simulation models for OTHR system, this option allows an approach to the actual behavior and a better estimation of the parameters that define the radio link.

The modeled transmission and reception processes in which the software is based are shown schematically in the block diagram of Fig. 2. The inputs of this software are transmitter and target geographic location and altitude, target orientation with respect to North, date and time, radar signal frequency, polarization, elevation angle, beam width, azimuth, transmitted power, transmitter and receiver gain, sea state, and target type. The outputs, which are the parameters needed to define radio link conditions, are maximum usable frequency (MUF), minimum usable frequency (LUF), maximum and minimum elevation angles, target radar cross section (RCS), noise power, clutter, Bragg frequency, attenuation, received power, group delay and ground range. In the following sub-sections, these together with models used in the different modules to define an OTH radio-link are described.

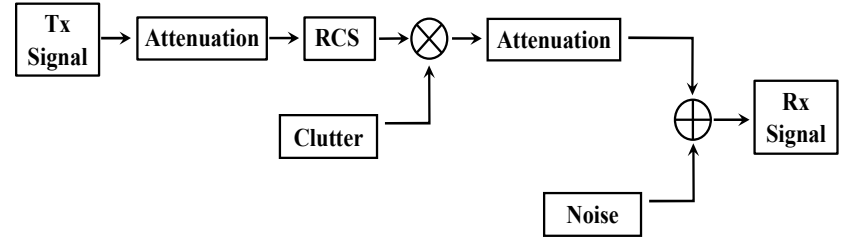

Fig. 2. Transmission and reception process block diagram. Each block modifies one or more radio wave parameters during its propagation.

\subsection{Elevation Angle}

The elevation angle $\theta$ is the angle between the transmitted signal and the plane tangent to Earth. For a given radar frequency, $f, \theta$ has a minimum $\theta_{\min }$ and a maximum value $\theta_{\max }$ up to which there is ionospheric reflection [7], [8]. $\theta_{\max }$ and $\theta_{\min }$ are the complementary angles to the critical incidence angles, so these can be determined through

$$
\begin{gathered}
\theta_{\max }=\frac{\pi}{2}-\cos ^{-1}\left(\frac{f o F 2}{f}\right), \\
\theta_{\min }=\frac{\pi}{2}-\sin ^{-1}\left(\frac{D}{D+h}\right)
\end{gathered}
$$

where $f_{O F}$ is the critical $F 2$ frequency, and it is obtained from the International Reference Ionosphere Model, IRI2012 [9], $D$ is the desired ground range, and $h$ is the virtual height of reflection obtained from the ray tracing module described ahead.

\subsection{Ray Path}

Key components of the radio wave ray path are ground range $D$, reflection height $h$ and group delay, which depend on the radio wave characteristics such as frequency, amplitude, and polarization, and in ionospheric conditions as well [7], [8]. Due to this dependence on the ionosphere these parameters strongly vary with local time, day of the year, geographic location, and solar activity. In addition, the overall propagation process in the ionosphere is a complex phenomenon.

A three-dimensional numerical ray tracing code [10] (Jones and Stephenson) based on Hamilton's equations is used to determine the radio wave path.

The IRI-2012 model [9] provides the ionospheric conditions necessary to run the ray tracing code. The key parameter here is the ionosphere refraction index which, neglecting electrons collision frequency and the Earth's magnetic field [7], is given by

$$
\mu=\sqrt{1-\frac{f o F 2^{2}}{f^{2}}} .
$$

There is a minimum frequency for which there is reflection from the ionosphere and also a maximum above which the radio wave passes through it. Therefore, there is a frequency range that is usable for an OTHR, and its extreme values are explained below.

\subsection{Maximum and Lowest Usable Frequencies}

The extreme frequency values correspond to the maximum and lowest usable frequencies, MUF and LUF respectively. For a given angle $\theta$ and $D$, MUF corresponds to the maximum frequency traveling in an oblique path that can be reflected back by the ionosphere for one single hop, 
which means, the ionosphere is transparent for higher frequencies. MUF is estimated from [7], [8]

$$
\operatorname{MUF}(D)=f o F 2 \sec (\theta) .
$$

The maximum frequency depends on geographic location and ionospheric characteristics. Unlike MUF that depends only on the transmitted signal and ionospheric conditions, LUF depends on the radar system characteristics, such as maximum transmitted power, $P_{\mathrm{T}}$, reception threshold, $U_{\mathrm{R}}$, attenuation during propagation and noise level. As a first approximation, a typical LUF value that is $\sim 5 \mathrm{MHz}$ for OTHR [8] can be considered.

\subsection{Radio Noise}

Noise is the unwanted electromagnetic energy that interferes with the radar ideal performance and may be superimposed on, or combined with, the wanted signal. It is typically represented as a random process [11], [12].

Noise determines the lowest echo intensity needed to be detected, and according to its origin, it can be classified into environmental and internal noise [12], [13]. The first one can be due to galactic cosmic rays, atmospheric and ionospheric disturbances, and/or human environmental sources, while the second one is due to internal radar circuit sources, mainly the antenna and receptor. Noise level varies all the time during the day, season, location and signal frequency. Typical environmental density noise is $\sim-175 \mathrm{dBW} / \mathrm{Hz}$, and internal noise is $\sim-195 \mathrm{dBW} / \mathrm{Hz}$ [2].

The noise figure $F_{\mathrm{n}}$ is determined following the information on the background levels of radio-frequency noise from Recommendation ITU-R P.372-12 [12].

The $F_{\mathrm{n}}$ of galactic noise is

$$
F_{\mathrm{n}}=52-23 \log (f) \text {. }
$$

The $F_{\mathrm{n}}$ of artificial and human noise is

$$
F_{\mathrm{n}}=c-d \log (f)
$$

where constants $c$ and $d$ are retrieved from ITU Recommendation [12].

The noise figure of atmospheric noise is obtained from tables and figures in ITU Recommendations [12].

If more than one kind of noise is present and if they are of comparable size, the Medium Noise Figure $\left(F_{\mathrm{m}}\right)$ is used. $F_{\mathrm{m}}$ is obtained as the sum of all the noise figures present [12].

\subsection{Clutter}

Clutter is the unwanted returns in the radar signals. The basic types of clutter can be summarized as surface and volume clutter. The first is due to ground and sea returns, and the second to chaff, rain, or birds for example [2], [13], [14].
In the case of sea clutter, the amount of clutter can be obtained by

$$
\sigma_{\mathrm{C}}=\sigma^{0} A
$$

where $\sigma_{\mathrm{C}}$ is the clutter radar cross section, $\sigma^{0}$ is the scattering coefficient, and $A$ is the area of scattering patch. The scattering coefficient of the clutter source has a PDF that determines its amplitude distribution. Even though $\sigma^{0}$ depends on the radio wave polarization, frequency, angle of incidence and sea surface conditions (or "roughness"), typical mean values obtained from tables are assumed [2]. Three different options for PDF are included: K-distribution, Lognormal, and Rayleigh type [15].

Sea clutter first order component, which is the one here considered, consists of two strong spectral lines known as 'Bragg lines'. These lines are due to resonant scattering of the transmitted radar signal by ocean waves that have a wavelength equal to one half of the radar wavelength. The frequency of the Bragg lines, $f_{\mathrm{B}}$, is given by

$$
f_{\mathrm{B}}= \pm \sqrt{\frac{g f}{2 \pi c}} \cong \pm 0.102 \sqrt{f(\mathrm{MHz})}
$$

where $g$ is the gravity acceleration and $c$ the light speed [2]. The Doppler spectrum of this phenomenon is shown in Fig. 3.

\subsection{Target Radar Cross Section}

The radar cross section (RCS) represents the target ability to reflect the incident radio wave in every direction. It depends on the shape, size and surface type of the target, and the frequency, polarization and incidence angle of the signal upon the target as well.

There are several methods to determine the RCS [16], [17]. In this work, the electromagnetic simulations software CST ( ${ }^{\circledR}$ Computer Simulation Technology) Microwave Studio was used to simulate RCS. The inputs needed

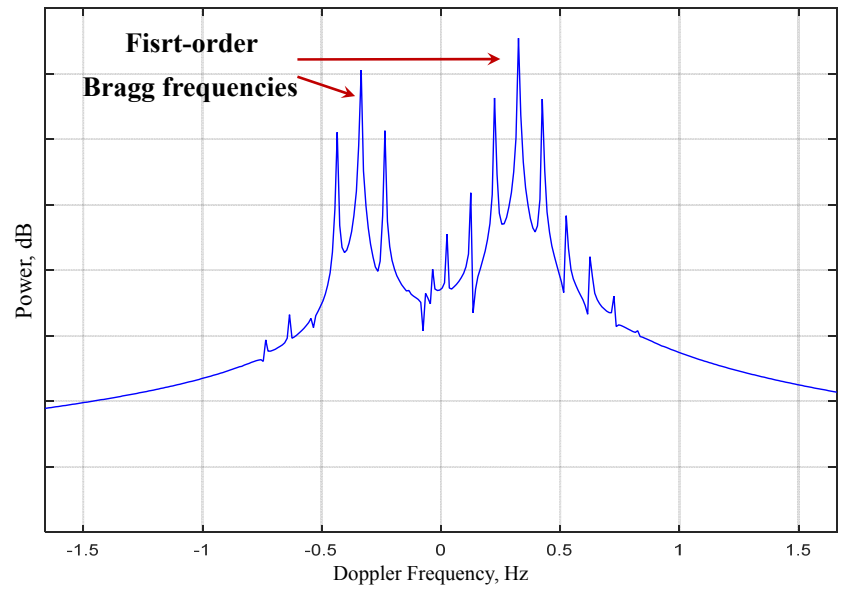

Fig. 3. Doppler spectrum of the received signal where the first-order Bragg frequencies are indicated with red arrows. 
are obtained from a 3-D CAD model, together with the materials of which the object is composed. The CST output is loaded then as an input for the RCS model. Simple CAD models can be used here since the results do not differ much from using complex ones in OTHR systems.

The simulation result of our model is target RCS in spherical coordinates for a given frequency and polarization of the radio wave incident.

\subsection{Attenuation}

Attenuation, or loss, is a reduction in power that results from absorption along the radio wave path or radar components. The most significant are geometric, deviative and non-deviative attenuations [8]. The first one, $L_{\mathrm{g}}$, corresponds to the loss of energy due to its distribution over the spherical surface and is estimated in $\mathrm{dB}$ from [8]

$$
L_{\mathrm{g}}=32.45+20 \log (R)+20 \log (f)
$$

where $R$ is the total path length covered by the ray and is obtained from the ray-tracing module.

The deviative attenuation, $L_{\mathrm{d}}$, is due to the portion of the radio path in the ionosphere close to the point of reflection. It is usually small and will be neglected here.

Non-deviative attenuation, $L_{\text {nd }}$, is mainly due to the ray path through the lowest ionospheric layers that $\mathrm{D}$ and $\mathrm{E}$ regions, and can be approximated in $\mathrm{dB} / \mathrm{km}$ by equation (20) in [20].

\subsection{Receiver Power}

The received power, $P_{\mathrm{R}}$, is the power returned to the radar and serves to determine its performance since it establishes a limit to its detection ability. In fact, the information in an OTHR (or any radar) is obtained by receiving and processing radio signals. $P_{\mathrm{R}}$ can be obtained from the radar equation [13], [14], which comes from the wellknown Friis equation [19], adapted to an OTHR

$$
P_{\mathrm{R}}=\frac{P_{\mathrm{T}} c^{2} G^{2} \sigma}{(4 \pi)^{3} R^{4} f^{2} L}
$$

where $P_{\mathrm{T}}$ is the transmitted power in Watts, $R$ the traveled distance in $\mathrm{km}, G_{\mathrm{T}}$ and $G_{\mathrm{R}}$ the transmitter and receiving antennas gain, which in our case are the same, $L$ the attenuation and $\sigma$ the RCS in square meter. In (10) the coherent processing interval $T$ for this first instance is not considered.

\subsection{Software Tool Implementation}

This software tool was written in Matlab for the low complexity calculation modules (Elevation angle, MUF, LUF, Noise, Clutter), and for the input and output routines, while the ray path module was written in Fortran. Jones and Stephenson ray tracing code here implemented and

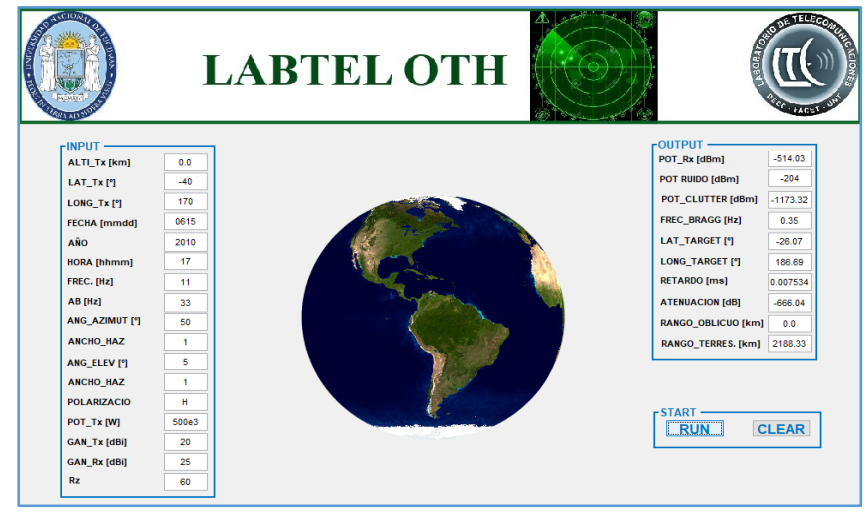

Fig. 4. Graphical interface. Left: data input, right: data output.

IRI-2012 model used to obtain the required ionospheric parameters, perform numerical calculations of high complexity which are faster and work optimally in Fortran.

The parameters that define the search scenario are entered through a graphic interface (Fig. 4).

A noteworthy aspect is the use of IRI model, which was modified by eliminating particular modules that are not used in our case (like for example the calculation of each ion species concentration) and by adding modules that allow the interaction between the ray tracing and IRI model. The new modules fulfill among other tasks: conversion of spherical to geographical coordinates, the retention and updating of the coordinates of the wave vector before and after each estimate of the next position, parameterize the IRI model with the input data selected by the user, calculate the critical frequency of the plasma from the value of the electron density in a specific coordinate, determine the new position of the wave vector by solving a system of differential equations based on the critical frequency of the plasma. The partial differential equations are solved numerically with a given step, and in each of them, the IRI model is called to obtain the corresponding ionospheric parameters to calculate the refractive index.

The time execution depends strongly on this step size. For a $10 \mathrm{~km}$ step and a Core I3 processor (or equivalent processor), the total execution time of this module is 10 minutes. This time can be highly improved by using an analytic ionospheric model like quasi-parabolic or Chapman layers, or by using a matrix of the ionospheric density in a gridded space.

The attenuation, noise and clutter modules are of low mathematic complexity, and most of the variables are obtained from graphical curves given in [12], [20]. These curves were digitized and incorporated as look-up tables. For example, IRI-2012 is also called by the attenuation module to obtain the critical frequency of the E layer used to estimate $L_{\text {nd. }}$.

With regard, to RCS determination, electromagnetic simulation software was adopted which allows discretization of the problem with the Method of Moments in the form of surface integrals combined with the Multi-Level Fast Multipole Method (MLFMM), where a surface mesh 
is used that generates fewer cells in the mesh than the common volume methods. The computational effort with MLFMM is effectively proportional to the size of the problem. The software delivers discrete RCS values that are stored within three-dimensional matrices (frequency, incidence angle, azimuth angle). The reading of these values is done according to frequency, polarization, and incidence and azimuth angles. For this reason, a binary search is used in 3D matrix and a 3D interpolation method for the missing data as a result of performed discretization.

On the other hand, three main difficulties were encountered when programming the software. One is the optimization of the execution time of ray tracing block where the longest execution time is presented in each call to the IRI-2012 model. For the optimization, we proceeded to configure the block only in the initial call and later only update the geographical coordinates. We also adopted an integration step of $10 \mathrm{~km}$ which was determined by taking an average relationship between the precision of the ray tracing versus the execution time. Another difficulty was the digitalization of curves and graphics used to determine different parameters needed as input data in the clutter, attenuation and noise modules, and then their conversion into look-up tables, what greatly facilitated the values search and reading. Our last challenge was obtaining the CAD files for a set of targets used by the RCS module.

\section{Results}

Figure 5 shows the block diagram of the software tool developed. It mainly consists of two steps. In the first one, the possible $f$ and $\theta$ values are assessed in terms of the transmitter and target locations. In the second, $f$ and $\theta$ are selected to establish radio-link and estimate the main parameters required to evaluate its performance.

Tables 1 and 2 present an example of a simulation for a given set of initial conditions. In these tables, all the output parameters are listed for which a search scenario will be determined in terms of ionospheric parameters, target type, and clutter and noise conditions. The user can then modify different radar parameters such as $P_{\mathrm{T}}, f, \theta$, and $G$ until approaching a desired radio link characteristic. For example, to increase the SCR (Signal to Clutter Relationship) and SNR (Signal to Noise Relationship) if the performance of target detection is unsatisfactory, or to change the radio wave frequency and the elevation angle-for illuminating the desired area. In addition, it can be easily assessed which parameters significantly modify some output parameters, obtaining a first estimate of the system sensitivity to certain input modifications.

On the other hand, we compared some of the outputs of the software tool here proposed with those obtained by Francis et al. [21]. In [21] ionospheric propagation is modeled using the ray-tracing toolbox PHaRLAP [22], with the ionospheric background obtained from IRI also. They present results using low and medium solar activity with levels ( $R z=20$ and 70, respectively). The ionospheric absorption model they use is from George and Bradley [23]. They assume a moving target (commercial aircraft), with typical RCS of $20 \mathrm{dBsm}$, and use a simplified clutter model with uniform backscatter coefficient, where the clutter is confined to Doppler band corresponding to no more than \pm 25 knots that is the minimum target velocity.

The properties of the signal are obtained from propagation tables over a range of environmental condition, parameterized by ground range and radar operating frequency, which are displayed as figures in [21]. We compared the received power values obtained with our software tool using border conditions given by Francis et al. [21], with the values read from their figures, for $20 \mathrm{se}-$ lected set of dates for 16:00 LT: winter low and medium solar activity, and summer medium solar activity. They correspond to the hypothetical New Zealand radar located at $41.15^{\circ} \mathrm{S}, 174.84^{\circ} \mathrm{E}$ considered in [21], operating in the band of 13 to $26 \mathrm{MHz}$, with a transmitter power of $40 \mathrm{~kW}$, oriented towards Sydney.

Figure 6 shows the scatter plot of the compared values. There is a good agreement taking into account the linear fit equation (with a slope very close to 1 and an intercept of $\sim 8 \mathrm{dBW}$ ), even though the dispersion (correlation coefficient $\sim 0.5$ ). The standard deviation, which is a measure of the average difference between the two sets of $P_{\mathrm{R}}$ values, is $\sim 10 \mathrm{dBW}$, which may be attributable mainly to the difference in models used for each module (ray path, clutter, RCS, attenuation). We also verify that there is no propagation support at all for frequencies above $10 \mathrm{MHz}$ with solar activity minimum in winter at 4:00 LT.

In conclusion, we consider the difference $\sim 10 \mathrm{~dB}$ as acceptable reminding that the main aim of this software tool is to provide a starting point for an OTH radar design, which has also other calibration tools, such as ionospheric sounders and HF receivers to determine the radio-electric spectrum among others. All together added to the first radar explorations they finally serve to optimize the OTH radar performance.

\section{Conclusion}

In the present work, a software tool is designed to estimate the main parameters in the transmission-reception process for an OTH radar design.

We consider that taking into account the care required in selecting radar operation parameters to achieve the best performance, a methodology for assessing the main parameters for a skywave OTH radar design, as the software tool here presented, is always useful and worthy.

This work's contribution is that through simulations, which allow to model and forecast the transmission-reception process in a quite realistic way, we can determine the main parameters of the OTH radar. These contributions are achieved through two aspects: methodology and software implementation. 
Regarding methodology, each block in the process of transmission and reception has a set of parameters which can be modified by the user, together with the radio link conditions. Unlike other simulation models, this option allows an approach to the actual behavior and a better estimation of the parameters that define the radio link.
Regarding software implementation, the modular approach of the software allows users to add new units or modify the existing ones independently of each other (e.g. including Traveling Ionosphere Disturbances (TIDs) and/or the Earth's magnetic field effect on propagation). In addition, the software tries to automate the entire process

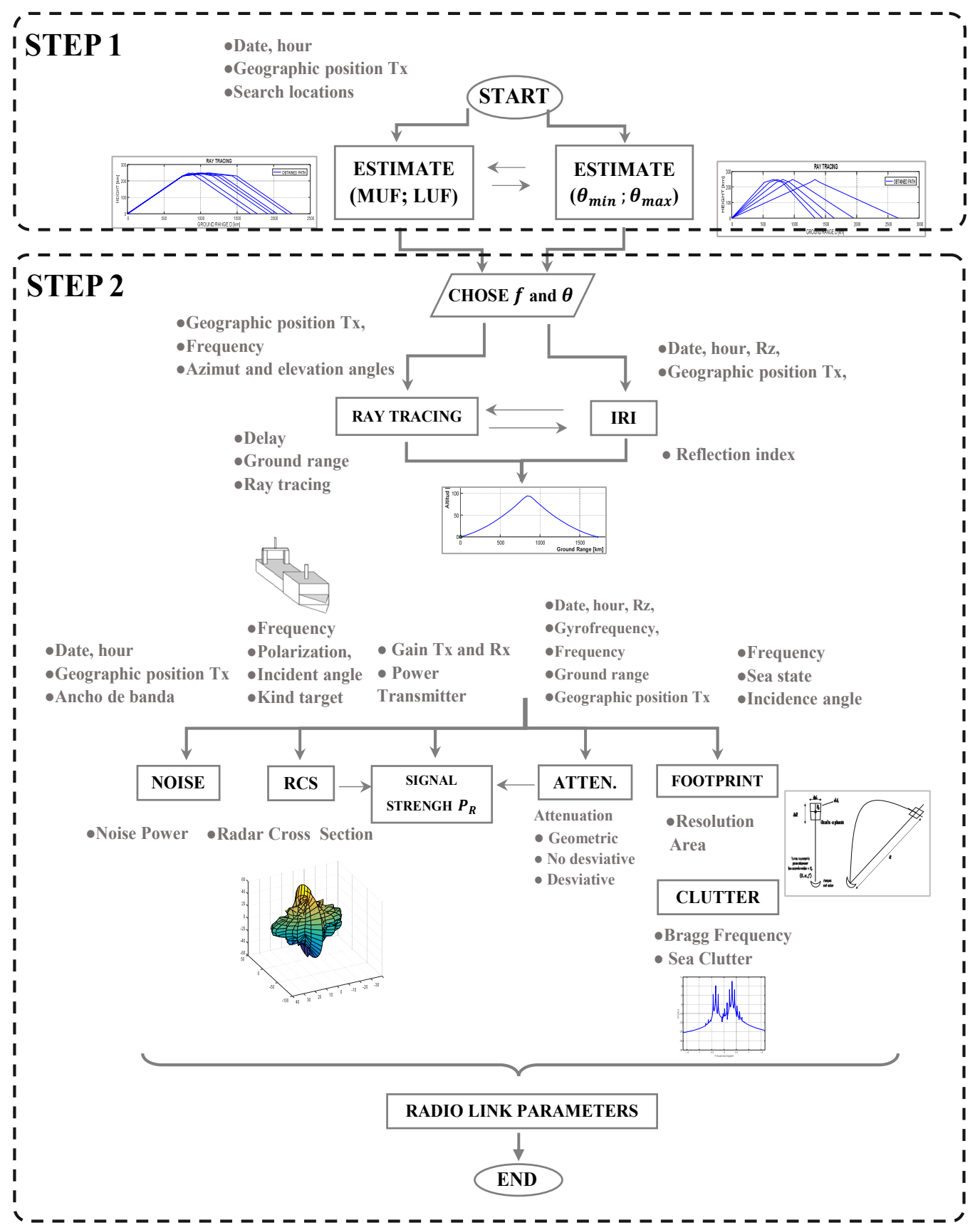

Fig. 5. Flowchart of software development.

\begin{tabular}{|c|c|c|c|}
\hline \multicolumn{2}{|c|}{ Input } & \multicolumn{2}{c|}{ Output } \\
\hline Parameter & Value & Parameter & Result \\
\hline Transmitter geographic location and altitude & Lat:48 N Long:80 W Alt: $0 \mathrm{~km}$ & MUF & $15 \mathrm{MHz}$ \\
\hline Target geographic location & Lat:45 N Long:101 W & LUF & $5 \mathrm{MHz}$ \\
\hline Target Orientation with respect to North & Azimuth: $10^{\circ}$ & Maximum elevation angle & $5^{\circ}$ \\
\hline Date and Time & $29 / 05 / 2000,12: 00 \mathrm{am}$ & Minimum elevation angle & $1^{\circ}$ \\
\hline
\end{tabular}

Tab. 1. Input parameters used in Step 1 which constitutes the initial conditions for the OTHR system, and first output set. 


\begin{tabular}{|c|c|c|c|}
\hline \multicolumn{2}{|c|}{ Input } & \multicolumn{2}{c|}{ Output } \\
\hline Parameter & Value & Parameter & Result \\
\hline Radar signal frequency & $12 \mathrm{MHz}$ & Target RCS & $29 \mathrm{dBsm}$ \\
\hline Polarization & Horizontal & Noise power & $-121 \mathrm{dBm}$ \\
\hline Beam width & $33 \mathrm{kHz}$ & Clutter power & $-232 \mathrm{dBm}$ \\
\hline Elevation angle & $3^{\circ}$ & Bragg Frequency & $\pm 0.36 \mathrm{~Hz}$ \\
\hline Beam width & $3^{\circ}$ & Attenuation Total & $-210 \mathrm{~dB}$ \\
\hline Azimuth & $25^{\circ}$ & Received power & $-263 \mathrm{dBm}$ \\
\hline Beam width & $3^{\circ}$ & Group delay & $5.8 \mathrm{~ms}$ \\
\hline Transmitter power & $500 \mathrm{~kW}$ & Ground range & $1630 \mathrm{~km}$ \\
\hline Transmitter and Receiver gain & $20 \mathrm{~dB}$ & & \\
\hline Sea state & State $3\left(\sigma^{0}=-30 \mathrm{~dB}\right)$ & & \\
\hline Target type & Offshore vessel & & \\
\hline
\end{tabular}

Tab. 2. Input parameters used in Step 2 based in Step 1 outputs (listed in Tab. 1), and outputs that constitutes the parameters that define the state of the radio link.

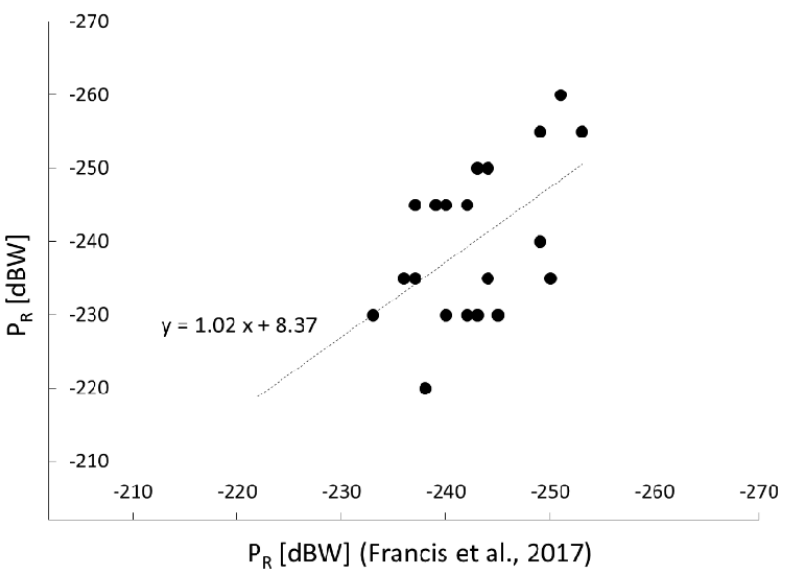

Fig. 6. Scatter plot of the received power estimated with the software tool presented here, $P_{\mathrm{R}}$, and $P_{\mathrm{R}}$ obtained from Francis et al. [19] tables. Linear fit (dashed line) and its equation.

of transmission and reception, freeing the user from the task of reading from tables or graphs some values of necessary parameters.

Furthermore, an advantage of this software tool is its versatility for different scenarios that can result from varying the target through its RCS, radar geographic location, surveillance area, time, and noise and clutter conditions, offering a fast tool and a simple way to evaluate the radar system sensibility to the input parameters in their respective range of variation. And finally, this tool can be considered a first step to assess the initial parameters in an $\mathrm{OTH}$ radar system design.

The future work will be to generate the received signal (time series) from the parameters that define it, followed by several methods and algorithm applications for target detection to obtain a tool that allows to design and test detection techniques.

\section{Acknowledgments}

We thank the CONICET (Argentina), by the Doctoral Scholarship of Zenon Saavedra, Universidad Nacional de
Tucumán (Argentina) and Agencia Nacional de Promoción Científica y Tecnológica (Argentina) for the grants through the Projects PICT2015-0511 (FONCyT-MINCyT), and PIUNT 26E/508 (UNT-Argentina).

\section{References}

[1] HEADRICK, J. M., SKOLNIK, M. I. Over-the-horizon radar in the HF band. Proceedings of the IEEE, 1974, vol. 62, no. 6, p. 664-673. DOI: 10.1109/PROC.1974.9506

[2] FABRIZIO, G. A. High Frequency Over-the-Horizon Radar. Fundamental Principles, Signal Processing, and Practical Applications. $1^{\text {st }}$ ed. New York (United States): McGraw Hill, 2013. ISBN: 978-0387231907

[3] HEADRICK, J. M., THOMASON, J. F. Applications of high-frequency radar. Radio Science, 1998, vol. 33, no. 4, p. 1045-1054. DOI: 10.1029/98RS01013

[4] KUSCHEL H., HECKENBACH J., MULLER S., et al. On the potentials of passive, multistatic, low frequency radars to counter stealth and detect low flying targets. In 2008 IEEE Radar Conference. Rome (Italy), 2008. DOI: 10.1109/RADAR.2008.4720984

[5] AZZARONe, A., BIANCHI, C., PEZZOPANE, M., et al. IONORT: A Windows software tool to calculate the HF ray tracing in the ionosphere. Computers and Geosciences, 2012, vol. 42, p. 57-63. DOI: 10.1016/j.cageo.2012.02.008

[6] NAGARAJOO, K. Ray tracing in realistic 3D ionospheric model. In Proceeding of the 2015 International Conference on Space Science and Communication (IconSpace). Langkawi (Malaysia), 2015, p. 267-272. DOI: 10.1109/IconSpace.2015.7283764

[7] DAVIS, K. Ionospheric Radio Propagation. Washington (USA): Dept. of Commerce, National Bureau of Standards, 1965. ISBN: 1124067051

[8] ZOLESI, B., CANDER, L. R. Ionospheric Prediction and Forecasting. Berlin (Germany): Springer, 2014. ISBN: 978-3-642$38429-5$

[9] BILITZA, D., ALTADILl, D., ZHANG, Y., et al. The International Reference Ionosphere 2012 - a model of international collaboration. Journal of Space Weather and Space Climate (SWSC), 2014, vol. 4, p. 1-12. DOI: 10.1051/swsc/2014004

[10] JONES, R. M., STEPHENSON, J. J. A versatile three-dimensional ray tracing computer program for radio waves in the ionosphere. OT Report, 75-76. Department of Commerce, Office of Telecommunication. Washington (USA): U.S. Government Printing Office, 1975. 
[11] ITU (International Telecommunication Union), Radiocommunication vocabulary, Recommendation ITU-R V.573-5. Geneva (Switzerland), 2007.

[12] ITU (International Telecommunication Union), Radio Noise, Recommendation ITU-R P.372-12. Geneva (Switzerland), 2015.

[13] SKOLNIK, M. I. Radar Handbook. 3rd ed. (USA): McGraw-Hill, 2008. ISBN: 978-0-07158942-0

[14] BARTON, D. K., LEONOV, S. A. (eds.) Radar Technology Encyclopedia. (USA): Artech House, 1998. ISBN 0-89006-893-3

[15] BILlingSLEY, J. B. Low-Angle Radar Land Clutter. Measurements and Empirical Models. New York (USA): William Andrew Publishing, 2002. ISBN: 1-891121-16-2

[16] DIAZ CHARRIS, V., GOMEZ TORRES, J. M. Analysis of radar cross section assessment methods and parameters affecting it for surface ships. Ship Science \& Technology, 2012, vol. 6, p. 91-106. DOI: $10.25043 / 19098642.72$

[17] EL-DARYMLI, K., GILL, E. W., MCGUIRE, P., et al. Automatic target recognition in synthetic aperture radar imagery: A state-ofthe-art review. IEEE Access, 2016, vol. 4, p. 6014-6058. DOI: 10.1109/ACCESS.2016.2611492

[18] DAVIES, K. Ionospheric Radio. London (UK): The Institution of Engineering and Technology, 1990. ISBN: $086341186 \mathrm{X}$

[19] FRIIS, H. T. A note on a simple transmission formula. Proceedings of the I.R.E., 1946, vol. 34, no. 5, p. 254-256. DOI: 10.1109/JRPROC.1946.234568

[20] ITU (International Telecommunication Union), Method for the Prediction of the Performance of HF Circuits ITU-R P.533-13. Geneva (Switzerland), 2015.

[21] FRANCIS, D. B., CERVERA, M. A., FRAZER, G. J. Performance prediction for design of a network of skywave over-the-horizon radars. IEEE Aerospace and Electronic Systems Magazine, 2017, vol. 32, p. 18-28. DOI: 10.1109/MAES.2017.170056

[22] PEDERICK, L. H., CERVERA, M. A. A directional HF noise model: Calibration and validation in the Australian region. Radio Science, 2016, vol. 51, p. 25-39. DOI: 10.1002/2015RS005842
[23] GEORGE, P. L., BRADLEY, P. A. A new method of predicting the ionospheric absorption of high frequency waves at oblique incidence. Telecommunication Journal, 1974, vol. 41, p. 307-311.

\section{About the Authors ...}

Zenon SAAVEDRA was born in Neuquen, Argentina. He received his Electronic Engineer degree in 2013, at the Universidad Nacional de Tucuman, Argentina. His research interests include radar systems development and design in general and aeronomy. $\mathrm{He}$ is a $\mathrm{PhD}$ student, and his thesis deals with OTH radar systems.

Julio N. ARGOTA was born in Tucuman, Argentina. He received his Electronic Engineer degree in 2017, at the Universidad Nacional de Tucuman, Argentina. His research interests include radar systems development and design in general and aeronomy. $\mathrm{He}$ is a $\mathrm{PhD}$ student, and his thesis is about NVIS communications.

Ana G. ELIAS was born in Tucuman, Argentina. She received her PhD in Physics in 1999, at the Universidad Nacional de Tucuman, Argentina. Her research interests include aeronomy, geomagnetism and long-term changes in the upper atmosphere.

Miguel A. CABRERA was born in Neuquen, Argentina. He received his $\mathrm{PhD}$ in Physics in 2003, at the Universidad Nacional de Tucuman, Argentina. He is the Dean of Facultad de Ciencias Exactas y Tecnologia, Universidad Nacional de Tucuman. 